\title{
Inquéritos antropométricos e alimentares na população brasileira: importante fonte de dados para o desenvolvimento de pesquisas
}

\author{
Anthropometric and dietary surveys of the Brazilian population: \\ an important source of data for conducting research
}

Naiara Sperandio ${ }^{1}$

Silvia Eloiza Priore ${ }^{2}$
${ }^{1}$ Curso de Nutrição, Universidade Federal do Rio de Janeiro. R. Professor Aloisio Teixeira Gomes da Silva 50, Granja dos Cavaleiros. 27930560 Macaé RJ Brasil. naiarasperandio@ yahoo.com.br

${ }^{2}$ Departamento de Nutrição,

Universidade Federal de

Viçosa. Viçosa MG Brasil.

\begin{abstract}
The scope of the current study was to analyze the methodological differences between Brazilian anthropometric and dietary surveys, which are classified as home, school and telephone surveys, as well as to reflect on the potential benefits and obstacles involved in the use of secondary data. The documents that are the focus of this study are official published reports of the surveys. The results indicated methodological differences in the assessment of food intake, anthropometry, frequency and form of access to the databases. The secondary data sources, which were previously limited to the dissemination of printed reports, were made available as micro-data in electronic databases. However, these databases are as yet not frequently consulted by health researchers due to the complexity of data extraction and analysis. For this reason, courses on how to develop skills to use these databases in graduate health courses should be included as they contain variables related to the health and nutrition status of the population, in addition to socioeconomic and demographic variables, which are not found in traditional health information systems. The conclusion drawn is that working with secondary data requires training and that the use of this source of information contributes to adequate planning in health.
\end{abstract}

Key words Nutritional surveys, Anthropometry, Health planning
Resumo Objetivou-se analisar as diferenças metodológicas dos inquéritos antropométricos e alimentares brasileiros classificados em domiciliares, escolares e telefônicos, e refletir sobre as potencialidades e os entraves envolvidos na utilização dos dados secundários dos mesmos. Os documentos que compuseram o corpus deste artigo foram relatórios oficiais publicados dos estudos. Os resultados retrataram diferenças metodológicas em relação à avaliação do consumo alimentar, à antropometria, à periodicidade e à forma de acesso as bases de dados. As fontes de dados secundários, que antes limitavam-se à divulgação de relatórios impressos, passaram a ser disponibilizadas sob a forma de microdados em bases eletrônicas. No entanto, essas bases são ainda pouco exploradas pelos pesquisadores da saúde, devido à complexidade de extração e de análise dos dados. Isso justifica a importância da inserção nos cursos de pós-graduação em saúde de disciplinas que promovam habilidades para uso dessas bases, que contêm variáveis sobre a situação de saúde e nutrição da população, além das socioeconômicas e demográficas não presentes nos sistemas tradicionais de informação em saúde. Conclui-se que o trabalho com dados secundários exige qualificação e o uso dessa fonte de informação contribui para adequado planejamento em saúde.

Palavras-chave Inquéritos nutricionais, Antropometria, Planejamento em saúde 


\section{Introdução}

Inquéritos populacionais que incluem variáveis antropométricas e de consumo alimentar são relevantes para avaliação e monitoramento das condições de saúde, alimentação e nutrição da população. Os resultados desses estudos constituem evidências científicas sólidas para definição de prioridades internacionais, nacionais e regionais, como também para tomada de decisão política ${ }^{1,2}$.

A concretização de estudos de avaliação do consumo alimentar, em nível nacional, é complexa, visto as dimensões territoriais do Brasil e suas diferenças sociais, regionais e culturais, sendo mais comum nos inquéritos a aferição de medidas antropométricas. No entanto, a análise representativa do consumo alimentar possibilita o conhecimento de hábitos, adequações e inadequações alimentares e nutricionais, assim como a própria interpretação dos dados antropométricos, o que reforça a importância de sua avaliação nos diferentes grupos etários ${ }^{1,2}$.

No Brasil, na década de 1970, realizou-se o primeiro inquérito nacional com informações acerca do consumo alimentar e estado nutricional dos brasileiros, denominado de Estudo Nacional de Despesa Familiar (ENDEF). O ENDEF utilizou a metodologia de pesagem direta dos alimentos pelo período de sete dias consecutivos. Tal procedimento possibilitou captar diferenças no consumo alimentar no decorrer da semana, inclusive variações típicas de fins de semana. Contudo, devido a limitações como custo elevado, alta complexidade, extenso período de coleta, interferência do pesquisador na rotina alimentar, dentre outros, não mais ocorreram avaliações periódicas do consumo alimentar, até a publicação da Pesquisa de Orçamento Familiar (POF) de 2008/09 13 .

As edições anteriores da POF (1987/88; 1995/96; 2002/03) trouxeram informações sobre a disponibilidade de alimentos para consumo nos domicílios ${ }^{5-7}$. A edição de 2008/09, além dos dados de disponibilidade, avaliou o consumo alimentar individual de uma subamostra da população ${ }^{8}$.

A POF, assim como os inquéritos descritos a seguir, são domiciliares, ou seja, a coleta dos dados acontece nos domicílios que são selecionados a partir de processo de amostragem. Dentre os inquéritos domiciliares brasileiros que disponibilizam informações acerca do consumo alimentar e/ou antropometria, destacam-se: a Pesquisa Nacional sobre Saúde e Nutrição (PNSN/1989)9 , realizada em única versão pelo Instituto Brasi- leiro de Geografia e Estatística (IBGE) em parceria com o Instituto Nacional de Alimentação e Nutrição (INAN), extinto em 1997, dando lugar para atual Coordenação Geral de Alimentação e Nutrição (CGAN); a Pesquisa sobre Padrões de Vida (PPV/1997) $)^{10}$, desenvolvida pelo IBGE em parceria com o Banco Mundial; a Pesquisa Nacional de Demografia e Saúde (PNDS de 1996 e 2006 $)^{11,12}$, promovida pelo Ministério da Saúde e o Centro Brasileiro de Análise e Planejamento (Cebrap); e a Pesquisa Nacional de Saúde $(\mathrm{PNS} / 2013)^{13}$, promovida pelo Ministério da Saúde em parceria com o IBGE.

A PNS terá periodicidade semelhante à da POF, isto é, cinco anos, e trata-se da primeira pesquisa do IBGE que realizou coleta de sangue para exames laboratoriais, a fim de caracterizar o perfil lipídico, de glicemia e de creatinina plasmática de um morador, com mais de 18 anos, em cada domicílio. A análise bioquímica somada à avaliação antropométrica, do consumo alimentar e dos dados socioeconômicos, possibilitaram avaliação completa do estado nutricional da população, assim como seus fatores determinantes ${ }^{13}$.

Além dos inquéritos domiciliares supracitados tem-se os escolares, como a Pesquisa Nacional de Saúde do Escolar (PNSE de 2009 e 2012) ${ }^{14,15}$ e o Estudo de Riscos Cardiovasculares em Adolescentes $(\text { ERICA } / 2014)^{16}$. No ERICA, semelhante à PNS do IBGE, também foi coletado amostras de sangue para exames laboratoriais, sendo dosados colesterol total, HDL-colesterol, triglicerídeos, glicose de jejum, hemoglobina glicada e insulina de jejum, de uma subamostra de 40 mil adolescentes $^{16}$.

Os inquéritos escolares fornecem informações importantes de dois públicos - escolares e adolescentes - que não possuem o hábito de procurar os serviços de saúde, a não ser em situações de ocorrência de agravos e doenças. Sendo assim, esses inquéritos colaboram para a definição de prioridades e ações de promoção da saúde e de prevenção de agravos direcionados para esses grupos, cujos dados são limitados em sistemas de informação tradicionais ${ }^{14-16}$.

Além dos inquéritos domiciliares e escolares tem-se os telefônicos, como é o caso da Vigilância dos Fatores de Risco e Proteção para Doenças Crônicas por Inquérito Telefônico (VIGITEL 2006; 2007; 2008; 2009; 2010; 2011; 2012; 2013 e 2014) ${ }^{17}$. O Vigitel, desde a sua primeira edição em 2006, vem sendo desenvolvido anualmente nas capitais dos estados e Distrito Federal, sendo o foco principal a vigilância e o monitoramento dos principais fatores de risco e de proteção 
para o desenvolvimento de doenças crônicas não transmissíveis, que constituem, atualmente, a principal causa de morbimortalidade no Brasil e demandam atenção especial na agenda política de alimentação e nutrição ${ }^{17}$.

Conforme descrito acima, é possível o desenvolvimento de pesquisas de relevância para a saúde coletiva a partir da utilização dos dados secundários, disponibilizados pelos inquéritos populacionais realizados no Brasil desde a década de 70. Contudo, alguns entraves ainda inviabilizam a utilização desses dados, principalmente os que envolvem a falta de informação sobre onde buscar as bases de dados, que são de acesso livre e gratuito, e de como extrair e proceder com as análises estatísticas, uma vez que o plano de amostragem dos inquéritos possuem certas particularidades, discutidas ao longo deste artigo, que os diferenciam do processo amostral comumente utilizado em pesquisas de menor abrangência ${ }^{18,19}$.

Diante disso, objetivou-se analisar as principais diferenças metodológicas dos inquéritos antropométricos e alimentares supracitados e refletir sobre as potencialidades, as dificuldades e os entraves envolvidos com a utilização dos dados secundários desses inquéritos.

\section{Métodos}

Os documentos que compuseram o corpus para realização deste artigo foram os relatórios oficiais sobre os inquéritos antropométricos e alimentares realizados no Brasil, acessados no site oficial do IBGE (http://www.ibge.gov.br/home/estatistica/pesquisas/pesquisas.php), do Ministério da Saúde (http://portalsaude.saude.gov.br/index. php/biblioteca) e do ERICA (http://www.erica. ufrj.br/). Além disso, também foram consultadas as bases de dados eletrônicas desses inquéritos. Destarte, inicialmente, buscou-se analisar os principais aspectos metodológicos dos estudos, enfatizando suas diferenças, principalmente, em relação ao tamanho amostral, à abrangência e aos métodos empregados para avaliação do consumo alimentar e antropometria. Em seguida, evoluiuse a discussão com reflexão acerca das potencialidades e dos entraves envolvidos com a utilização dos dados secundários disponibilizados por esses inquéritos.

\section{Resultados e discussões}

\section{Os principais inquéritos populacionais brasileiros em alimentação e nutrição e suas diferenças metodológicas}

O Quadro 1 sumariza as principais diferenças dos inquéritos populacionais brasileiros, citados na seção inicial deste artigo, que fornecem informações acerca das características alimentares e nutricionais da população, além de outras, como as socioeconômicas, as demográficas e as de estilo de vida.

No que diz respeito aos aspectos metodológicos, de maneira geral, tratam-se de estudos observacionais transversais, com delineamento complexo para determinação representativa da amostra. As principais diferenças relacionam-se ao tamanho amostral, à periodicidade de realização, às técnicas de avaliação antropométrica e de consumo alimentar, assim como às medidas aferidas que envolvem além do peso e da estatura, obtidas em praticamente todos os inquéritos, o perímetro do braço ${ }^{4}$ e da cintura ${ }^{12,13,16}$.

Em relação às características da amostra, alguns abrangeram todos os ciclos de vida ${ }^{4-10}$, outros apenas grupos etários específicos, como materno-infantil ${ }^{11,12}$, escolares ${ }^{14,15}$, adolescentes ${ }^{16}$ e adultos ${ }^{13,17}$.

Para a avaliação do consumo alimentar, alguns estudos analisaram efetivamente o consumo, através de métodos como pesagem direta de alimentos ${ }^{4}$, questionário de frequência de consumo alimentar ${ }^{10,12}$, registro alimentar ${ }^{8}$ e recordatório de 24 horas $^{16}$.

Em outros inquéritos a análise do consumo envolveu a avaliação da disponibilidade de alimentos adquiridos pelas famílias ${ }^{5-8}$, outra maneira empregada foi o questionamento sobre o consumo de determinados alimentos e/ou grupos, considerados marcadores saudáveis e não saudáveis da alimentação ${ }^{13-17}$. Esse tipo de análise não informa o consumo individual efetivo ou a distribuição intrafamiliar dos alimentos, sendo possível inferir sobre hábitos alimentares que podem constituir fatores de risco ou de proteção para o desenvolvimento de doenças.

A avaliação do consumo alimentar possibilita investigar a relação da alimentação com a ocorrência de determinados desfechos, como a desnutrição, sobrepeso e/ou obesidade, além da identificação de padrões alimentares que, se analisados com certa periodicidade, possibilitam traçar tendências das condições alimentares e nutricionais, o que pode auxiliar a elaboração e 
Quadro 1. Síntese dos principais inquéritos antropométricos e alimentares da população brasileira.

\begin{tabular}{|c|c|c|c|c|}
\hline Pesquisa & Amostra & Objetivos & $\begin{array}{l}\text { Consumo } \\
\text { alimentar }\end{array}$ & Antropometria \\
\hline $\begin{array}{l}\text { Estudo Nacional } \\
\text { de Despesas } \\
\text { Familiares } \\
(\text { ENDEF - 1974) }\end{array}$ & $\begin{array}{l}55 \text { mil domicílios; } \\
267.446 \text { pessoas. }\end{array}$ & $\begin{array}{l}\text { Avaliar as condições de vida } \\
\text { e a situação nutricional da } \\
\text { população. }\end{array}$ & $\begin{array}{l}\text { Pesagem } \\
\text { direta de } \\
\text { alimentos } \\
\text { durante } \\
\text { sete dias } \\
\text { consecutivos. }\end{array}$ & $\begin{array}{l}\text { Peso }(\mathrm{kg}) \\
\text { Altura }(\mathrm{cm}) \\
\text { Perímetro do } \\
\text { Braço }(\mathrm{cm})\end{array}$ \\
\hline $\begin{array}{l}\text { Pesquisa de } \\
\text { Orçamento } \\
\text { Familiar (POF) } \\
\text { de } 1987^{5} \text { e de } \\
1995^{6} .\end{array}$ & $\begin{array}{l}\text { 1987: } 13.707 \\
\text { domicílios. } \\
\text { 1995: } 19.816 \\
\text { domicílios. }\end{array}$ & $\begin{array}{l}\text { Traçar o perfil das condições } \\
\text { de vida da população a partir } \\
\text { da análise de seus orçamentos } \\
\text { domésticos. }\end{array}$ & $\begin{array}{l}\text { Aquisição } \\
\text { domiciliar de } \\
\text { alimentos. }\end{array}$ & $\begin{array}{l}\text { Não foi } \\
\text { avaliado. }\end{array}$ \\
\hline $\begin{array}{l}\text { Pesquisa } \\
\text { Nacional de } \\
\text { Saúde e Nutrição } \\
(\text { PNSN - 1989) }\end{array}$ & $\begin{array}{l}14.455 \text { domicílios; } \\
63.213 \text { indivíduos. }\end{array}$ & $\begin{array}{l}\text { Descrever o estado nutricional } \\
\text { da população brasileira, } \\
\text { além de caracterizar as } \\
\text { condições de saúde e a } \\
\text { estrutura socioeconômica nos } \\
\text { domicílios. }\end{array}$ & $\begin{array}{l}\text { Não foi } \\
\text { avaliado. }\end{array}$ & $\begin{array}{l}\text { Peso }(\mathrm{kg}) \\
\text { Altura }(\mathrm{cm})\end{array}$ \\
\hline $\begin{array}{l}\text { Pesquisa sobre } \\
\text { Padrões de Vida } \\
(\text { PPV }-1997)^{10}\end{array}$ & $\begin{array}{l}\text { Nordeste e } \\
\text { Sudeste. } \\
5.000 \text { domicílios. }\end{array}$ & $\begin{array}{l}\text { Identificar panorama do bem } \\
\text { estar social da população, } \\
\text { assim como os fatores } \\
\text { determinantes. }\end{array}$ & $\begin{array}{l}\text { Questionário } \\
\text { de frequência } \\
\text { com } 28 \text { tipos } \\
\text { de alimentos }\end{array}$ & $\begin{array}{l}\text { Peso }(\mathrm{kg}) \\
\text { Altura }(\mathrm{cm})\end{array}$ \\
\hline $\begin{array}{l}\text { Pesquisa } \\
\text { Nacional de } \\
\text { Demografia e } \\
\text { Saúde (PNDS de } \\
1996 \text { e 2006) }{ }^{11,12}\end{array}$ & $\begin{array}{l}\text { 1996: } 13.283 \\
\text { domicílios; } 12.612 \\
\text { mulheres, } 2.949 \\
\text { homens e } 4.782 \\
\text { crianças menores } \\
\text { de } 5 \text { anos. } \\
\text { 2006: } 14.617 \\
\text { domicílios; } 15.575 \\
\text { mulheres e } 5.461 \\
\text { crianças menores } \\
\text { de } 5 \text { anos }\end{array}$ & $\begin{array}{l}\text { Caracterizar a população } \\
\text { feminina em idade fértil } \\
\text { e as crianças abaixo de } 5 \\
\text { anos de idade, segundo } \\
\text { fatores demográficos, } \\
\text { socioeconômicos e culturais. }\end{array}$ & $\begin{array}{l}\text { 2006: } \\
\text { Questionário } \\
\text { de frequência } \\
\text { com } 20 \\
\text { tipos de } \\
\text { alimentos } \\
\text { b. }\end{array}$ & $\begin{array}{l}\text { 1996: } \\
\text { Peso }(\mathrm{kg}) \\
\text { Altura }(\mathrm{cm}) \\
\text { 2006: } \\
\text { Peso }(\mathrm{kg}) \\
\text { Altura }(\mathrm{cm}) \\
\text { Perímetro da } \\
\text { cintura (cm) } \\
\text { das mulheres. }\end{array}$ \\
\hline POF - 2002/03 & $\begin{array}{l}48.470 \text { domicílios; } \\
182.333 \text { pessoas. }\end{array}$ & $\begin{array}{l}\text { Fornecer informações sobre a } \\
\text { composição dos orçamentos } \\
\text { domésticos e as condições } \\
\text { alimentares e nutricionais da } \\
\text { população. }\end{array}$ & $\begin{array}{l}\text { Aquisição } \\
\text { domiciliar de } \\
\text { alimentos. }\end{array}$ & $\begin{array}{l}\text { Peso }(\mathrm{kg}) \\
\text { Altura }(\mathrm{cm})\end{array}$ \\
\hline POF $-2008 / 09^{8}$ & $\begin{array}{l}55.970 \text { domicílios; } \\
159.941 \text { pessoas. } \\
\text { Análise do } \\
\text { consumo } \\
\text { alimentar: } 34.003 \\
\text { pessoas. }\end{array}$ & Idem POF 2002-2003. & $\begin{array}{l}\text { Aquisição } \\
\text { domiciliar de } \\
\text { alimentos. } \\
\text { Moradores } \\
\text { com mais } \\
\text { de } 10 \text { anos } \\
\text { preencheram } \\
2 \text { registros } \\
\text { alimentares } \\
\text { em dias não } \\
\text { consecutivos. }\end{array}$ & $\begin{array}{l}\text { Peso }(\mathrm{kg}) \\
\text { Altura }(\mathrm{cm})\end{array}$ \\
\hline
\end{tabular}


Quadro 1. continuação

\begin{tabular}{|c|c|c|c|c|}
\hline Pesquisa & Amostra & Objetivos & $\begin{array}{l}\text { Consumo } \\
\text { alimentar }\end{array}$ & Antropometria \\
\hline $\begin{array}{l}\text { Pesquisa } \\
\text { Nacional de } \\
\text { Saúde (PNS - } \\
2013)^{13}\end{array}$ & 62.986 domicílios. & $\begin{array}{l}\text { Coletar dados sobre a situação } \\
\text { de saúde e os estilos de vida } \\
\text { da população brasileira. }\end{array}$ & $\begin{array}{l}\text { Marcadores } \\
\text { positivos e } \\
\text { negativos } \\
\text { do consumo } \\
\text { alimentar } \\
\text { (questões } \\
\text { sobre o } \\
\text { consumo de } \\
\text { determinados } \\
\text { alimentos)c. }\end{array}$ & $\begin{array}{l}\text { Peso }(\mathrm{kg}) \\
\text { Altura }(\mathrm{cm}) \\
\text { Perímetro da } \\
\text { Cintura }(\mathrm{cm})\end{array}$ \\
\hline $\begin{array}{l}\text { Pesquisa } \\
\text { Nacional de } \\
\text { Saúde do Escolar } \\
(\text { PNSE de } 2009 \text { e } \\
\text { 2012) })^{14,15}\end{array}$ & $\begin{array}{l}\text { Escolares do } 9^{\circ} \\
\text { ano do Ensino } \\
\text { Fundamental de } \\
\text { escolas públicas } \\
\text { e privadas das } \\
\text { capitais brasileiras } \\
\text { e do Distrito } \\
\text { Federal. } \\
\text { 2009: } 6.780 \\
\text { escolas; } 60.973 \\
\text { escolares. } \\
\text { 2012: } 42.717 \\
\text { escolas; } 109.104 \\
\text { escolares. }\end{array}$ & $\begin{array}{l}\text { Conhecer a prevalência dos } \\
\text { fatores de risco e a proteção } \\
\text { à saúde dos adolescentes, e } \\
\text { orientar intervenções em } \\
\text { saúde adequadas. }\end{array}$ & $\begin{array}{l}\text { Marcadores } \\
\text { de } \\
\text { alimentação } \\
\text { saudável } \\
\text { e de não } \\
\text { saudáveld. }\end{array}$ & $\begin{array}{l}\text { 2009: } \\
\text { Peso }(\mathrm{kg}) \\
\text { Altura }(\mathrm{cm}) \\
\text { 2012: sem } \\
\text { antropometria. }\end{array}$ \\
\hline $\begin{array}{l}\text { Estudo de Riscos } \\
\text { Cardiovasculares } \\
\text { em Adolescentes } \\
(\text { ERICA/2014) })^{16}\end{array}$ & $\begin{array}{l}\text { Adolescentes de } 12 \\
\text { a } 17 \text { anos. } \\
\text { Total de } 1.251 \\
\text { escolas; } 75 \text { mil } \\
\text { estudantes. }\end{array}$ & $\begin{array}{l}\text { Conhecer a proporção de } \\
\text { adolescentes com diabetes } \\
\text { mellitus e obesidade, assim } \\
\text { como traçar o perfil dos } \\
\text { fatores de risco de doenças } \\
\text { cardiovasculares. }\end{array}$ & $\begin{array}{l}\text { Recordatório } \\
\text { de } 24 \text { horase. }\end{array}$ & $\begin{array}{l}\text { Peso }(\mathrm{kg}) \\
\text { Altura }(\mathrm{cm}) \\
\text { Perímetro da } \\
\text { cintura }(\mathrm{cm})\end{array}$ \\
\hline $\begin{array}{l}\text { Vigilância dos } \\
\text { fatores de risco } \\
\text { e proteção para } \\
\text { doenças crônicas } \\
\text { por inquérito } \\
\text { telefônico } \\
(\text { Vigitel 2006; } \\
2014)^{17}\end{array}$ & $\begin{array}{l}\text { Adultos com } \\
\text { mais de } 18 \text { anos } \\
\text { residentes nas } \\
\text { capitais dos } \\
26 \text { estados e } \\
\text { Distrito Federal. } \\
\text { Entrevistas } \\
\text { por inquérito } \\
\text { telefônico. }\end{array}$ & $\begin{array}{l}\text { Monitorar a magnitude } \\
\text { das doenças e dos agravos } \\
\text { não transmissíveis e de seus } \\
\text { determinantes. }\end{array}$ & $\begin{array}{l}\text { Marcadores } \\
\text { positivos e } \\
\text { negativos } \\
\text { do consumo } \\
\text { alimentarf. }\end{array}$ & $\begin{array}{l}\text { Dados } \\
\text { autodeclarados } \\
\text { de peso e } \\
\text { altura. }\end{array}$ \\
\hline
\end{tabular}

Fonte: Elaborado pelas autoras.

Nota: ${ }^{\text {a }} \mathrm{O}$ questionário foi aplicado a um responsável pelo domicílio e refletiu o consumo familiar dos alimentos. ${ }^{\mathrm{b}}$ Na PNDS o questionário de frequência referiu-se ao consumo de alimentos das crianças menores de cinco anos. Esse questionário foi respondido pela mãe, sendo o período de referência os sete dias anteriores a coleta. ${ }^{c}$ Foram considerados marcadores positivos da alimentação: consumo recomendado de frutas, legumes e verduras, e o consumo regular de feijão; os negativos foram: o consumo regular de refrigerantes, de leite integral, de carnes com excesso de gordura (gordura aparente e frango com pele) e o consumo de sal. As medidas antropométricas, assim como as informações sobre o consumo, foram determinadas apenas para um morador do domicílio acima de 18 anos. ${ }^{\mathrm{d}}$ Foram considerados marcadores de alimentação saudável o consumo de legumes e verduras, frutas e leite, em cinco ou mais dias na semana, e o de não saudáveis, o consumo de embutidos, biscoitos e refrigerantes, em cinco ou mais dias na semana. ${ }^{e}$ Foram aplicados dois recordatório de 24 horas, sendo que o primeiro envolveu todos os adolescentes, e o segundo apenas uma subamostra. Um programa computacional, para entrada de dados do recordatório alimentar de 24 horas, foi desenvolvido pela equipe do ERICA, e é de livre acesso para outros pesquisadores (http://www.erica.ufrj.br/programa-rec24h). ${ }^{\mathrm{f}}$ Questiona-se informações sobre o consumo regular de feijão, o hábito de consumo de carnes com excesso de gordura, hábito de consumo de leite integral, o consumo regular de refrigerantes, o consumo regular de frutas, legumes e verduras e o consumo adequado de frutas, legumes e verduras. 
a atualização de guias alimentares, assim como o planejamento e a avaliação de políticas públicas e programas de intervenção $0^{2,20}$.

Os resultados da POF 2008/09, acerca das características do consumo alimentar de brasileiros com mais de dez anos, contribuíram para a atualização do Guia Alimentar para População Brasileira, com a primeira edição publicada em 2006 e reedição em $2014^{21}$.

Em relação aos métodos de investigação do consumo alimentar, destaca-se que não existe um considerado padrão-ouro, todos (recordatório de 24 horas, registro alimentar, questionário de frequência de consumo alimentar, pesagem direta de alimentos, história dietética, dentre outros) possuem vantagens e limitações que devem ser analisadas, sendo que a escolha de qual método adotar vai depender das características da população a ser estudada e dos objetivos do estudo ${ }^{2,20}$.

No caso específico da POF 2008/09 a escolha do registro alimentar deu-se principalmente por ser o melhor que se adaptava à prática de coleta de dados da pesquisa (que se baseia no registro de informações pelos indivíduos em uma caderneta $)^{8}$.

A avaliação do consumo alimentar individual efetivo, apesar da importância, é onerosa e complexa, por conseguinte é mais comum nos inquéritos a análise da disponibilidade de alimentos para consumo ${ }^{18}$. Avaliações periódicas da disponibilidade de alimentos fornecem informações sobre tendências alimentares sendo que os resultados no Brasil têm indicado redução da aquisição de alimentos tradicionais, como arroz e feijão, e aumento da aquisição de alimentos processados e ultraprocessados ${ }^{22,23}$, cuja moderação de consumo foram ressaltadas no Guia Alimentar de 2014.

Modificações do hábito alimentar dos brasileiros, como o aumento da realização de refeições fora de casa, limitam a utilização de dados sobre a aquisição de alimentos para consumo dentro do domicílio, isso acaba por influenciar as metodologias dos inquéritos ${ }^{23,24}$. Na POF $2002 / 03$ os indivíduos registraram as despesas com alimentação realizadas fora do domicílio, o que reforçou a necessidade da avaliação do consumo alimentar individual efetivo (dentro e fora de casa) que ocorreu na edição posterior da pesquisa em 2008/0924.

A avaliação antropométrica, diferente do consumo alimentar, é considerada método direto de diagnóstico do estado nutricional. Nos inquéritos populacionais, as medidas antropométricas, especialmente o peso e a estatura, são amplamente utilizadas em função do baixo custo, da facilidade de treinamento pessoal para coleta dos dados e do tempo reduzido para aferição das medidas $^{25}$.

Entretanto, a validade e a precisão de um inquérito antropométrico depende do adequado treinamento da equipe de campo, da utilização de equipamentos calibrados e da padronização para aferição das medidas. Além disso, a avaliação temporal do estado nutricional, com base na análise de dados de diferentes inquéritos populacionais, torna necessário a padronização das referências antropométricas e dos pontos de corte utilizados para diagnóstico do estado nutricional ${ }^{25,26}$.

Estudos realizados na década de 70, 80 e 90 empregaram como referências antropométricas, para diagnóstico nutricional das crianças e dos adolescentes, curvas de crescimento diferentes das atuais recomendações do Ministério da Saúde, que são as da Organização Mundial da Saúde (OMS) de 2005, para crianças de 0 a 5 anos, e de 2007, para crianças e adolescente de 6 a 19 anos. Essas referências passaram a ser preconizadas, a partir de 2006, e foram adotadas pelos inquéritos mais atuais ${ }^{27}$.

No Vigitel, as medidas de peso e de altura foram autodeclaradas, sendo que nos demais inquéritos, reunidos no Quadro 1, essas medidas antropométricas foram aferidas pelos pesquisadores de campo previamente treinados. Um estudo que comparou medidas antropométricas declaradas com as obtidas de forma direta, encontrou boa concordância do diagnóstico nutricional, determinado pelas duas metodologias, desse modo os autores reforçaram a boa confiabilidade das medidas declaradas ${ }^{25}$.

Comparando os dados da prevalência de excesso de peso em adultos, da POF 2008/09 (cuja as medidas foram aferidas) $(49 \%)^{8}$, com a do Vigitel de 2009 (cuja as medidas foram autorreferidas) $(46 \%)^{17}$ observa-se que os resultados de prevalência foram próximos.

É relevante a ressalva de como as medidas antropométricas, nos estudos brasileiros, modificaram-se conforme a alteração do perfil epidemiológico da população. $\mathrm{O} \mathrm{ENDEF}{ }^{4}$, realizado na década de 70, além das medidas de peso e estatura, incluiu o perímetro do braço, um indicador utilizado para caracterização de perda de massa muscular. Nessa época, acreditava-se que a desnutrição no Brasil era de origem proteica, o que não foi confirmado pelos resultados que denunciaram ocorrência de desnutrição calórica ${ }^{27}$. Em contrapartida, os inquéritos mais recentes ${ }^{12,13,16}$ 
incluíram a medida de perímetro da cintura, que é um preditor de risco para doenças crônicas, como as cardiovasculares, um dos principais problemas de saúde pública no cenário atual ${ }^{27}$.

As diferenças metodológicas devem ser ressalvadas, se o propósito do pesquisador envolver a utilização de dados secundários de diferentes inquéritos.

Além dos dados antropométricos e de consumo alimentar, os inquéritos analisados (Quadro 1) também fornecem outras variáveis, como, por exemplo, as demográficas, as socioeconômicas e as de estilo de vida. A utilização dessas informações contribuem para análise mais completa e recortes por sexo, idade, faixas de rendimento e escolaridade, que possibilitam a determinação social do estado nutricional que envolve múltiplos condicionantes, para além dos nutricionais. Isso reforça a importância da realização dos inquéritos populacionais, uma vez que os sistemas de informação em saúde disponíveis (Sistema de Informações sobre Mortalidade; Sistema de Informações de Nascidos Vivos; Sistema de Informação de Agravos de Notificação; Sistema de Vigilância Alimentar e Nutricional, dentre outros) não disponibilizam número tão amplo de variáveis, como as fornecidas pelos inquéritos ${ }^{28}$.

Os inquéritos populacionais brasileiros constituem potenciais fontes de dados secundários para realização de pesquisas com diferentes objetivos, que podem abranger o diagnóstico e o monitoramento das condições alimentares e nutricionais da população, assim como a avaliação da eficácia, eficiência e efetividade de políticas públicas $^{29}$.

A vigilância alimentar e nutricional, compreendida sob uma ótica ampliada, conforme estabelecido na Política Nacional de Alimentação e Nutrição (PNAN), entende que a determinação da situação de saúde da população, deve envolver diferentes fontes de informação, dentre elas os inquéritos populacionais. A utilização desses dados em estudos é importante para a organização da atenção nutricional e a garantia do cuidado integral à saúde individual e coletiva ${ }^{30}$.

Contudo, a utilização dos dados secundários, dos inquéritos populacionais brasileiros, ainda é modesta, frente a potencialidade de desenvolvimento de investigações nos mais diferentes campos científicos.

\section{Acesso e utilização de dados secundários para desenvolvimento de pesquisas científicas}

Dentre as vantagens do uso de bancos de dados existentes, destacam-se: o baixo custo, o acesso via sistemas computadorizados de qualquer lugar e a disponibilidade de informações por longo período de tempo, o que permite avaliar tendência secular das condições de alimentação e nutrição de grupos populacionais ${ }^{31}$.

Para o acesso aos microdados dos estudos supracitados (Quadro 1) deve-se buscar orientações nos sites oficiais da instituição ou agência responsável pela realização da pesquisa. No caso dos estudos realizados pelo IBGE o acesso é livre e gratuito. Para extração dos dados deve-se selecionar o estudo desejado no site do IBGE (http:// www.ibge.gov.br/home/), onde estão listadas todas as pesquisas desenvolvidas e as que estão em desenvolvimento. Em relação à PNS de 2014 existe outra possibilidade, além do site do IBGE, para acesso aos microdados que é o site criado exclusivamente para essa pesquisa (http://www. pns.icict.fiocruz.br/index.php?pag=resultados).

$\mathrm{O}$ acesso aos dados dos estudos desenvolvidos pelo Ministério da Saúde vai depender da pesquisa. Para a PNDS tem-se disponível uma página oficial para divulgação dos resultados e dos microdados (http://bvsms.saude.gov.br/bvs/ pnds/banco_dados.php), já em relação ao Vigitel o acesso é através da base de dados da Secretária de Vigilância em Saúde (http://tabnet.datasus. gov.br/cgi/vigitel/vigteldescr.htm).

Em relação ao ERICA, a instituição coordenadora é a Universidade Federal do Rio de Janeiro (UFRJ) e para o acesso aos dados do estudo deve-se entrar em contato com os coordenadores (http://www.erica.ufrj.br) e enviar a proposta de pesquisa que se pretende desenvolver com a utilização dos dados do ERICA.

Os avanços tecnológicos impactaram de maneira marcante o desenvolvimento e a realização de pesquisas em saúde, alimentação e nutrição. Esses avanços foram importantes tanto para pesquisas baseadas em dados primários como para aquelas que utilizam dados secundários, tendo nesse último caso impacto mais significativo ${ }^{31,32}$.

As fontes de dados secundários no Brasil, que antes se limitavam à divulgação de relatórios im- 
pressos com informações agregadas, passaram a ser disponibilizadas sob a forma de microdados, em bases eletrônicas, que possibilitam maior flexibilidade e possibilidade de desenvolvimento de hipóteses e análises estatísticas diversificadas ${ }^{31,32}$.

O uso de dados secundários dos estudos epidemiológicos possui a vantagem da representatividade nacional das amostras, que podem ser estratificadas para nível regional ou estadual. Porém, vale destacar que nem todos os estudos têm cobertura nacional, e a realização de pesquisas a partir do uso dos microdados fica restrita a disponibilidade de variáveis investigadas nos inquéritos ${ }^{31,32}$.

Os inquéritos populacionais com representatividade nacional não possibilitam desagregações das informações para níveis locais. Uma alternativa, para acessar dados de representatividade local, é a utilização dos sistemas de informação em saúde, que, no caso específico de indicadores alimentares e nutricionais, seria o SISVAN $^{30}$. A partir da utilização dos dados do SISVAN, assim como de outros sistemas de saúde disponíveis, pode-se desenvolver pesquisas que retratam as condições alimentares e nutricionais da população e os seus determinantes em nível local.

Outro aspecto importante na utilização de dados secundários refere-se à qualidade e ao rigor metodológico envolvido com a coleta dos dados. Alguns problemas são comuns em estudos domiciliares, como a não resposta e valores de variáveis - como peso e estatura - considerados implausíveis. Dessa forma, antes da extração e análise dos dados deve-se consultar os documentos que detalham os aspectos metodológicos das pesquisas e ratificar se ocorreram procedimentos de crítica e de imputação, que garantem maior rigor metodológico e confiabilidade dos dados disponibilizados ${ }^{19,32}$.

Para exemplificar, na POF 2008/09 a proporção de valores implausíveis, para a medida de estatura de crianças menores de cinco anos, foi superior ao aceitável pela OMS (que considera como biologicamente implausíveis afastamentos maiores que seis desvios-padrão da distribuição de referência para a altura e cinco para o peso ou IMC; em inquéritos antropométricos de boa qualidade, a proporção de valores implausíveis não deve ser superior a $1 \%$ do total de pessoas avaliadas $)^{8}$. Sendo assim, o pesquisador que utilizar os microdados da $\mathrm{POF}$, referentes à variável estatura de menores de cinco anos, deve fazer uma ressalva em relação à precisão insuficiente na mensuração dessa medida.

A extração e a utilização dos microdados disponibilizados nas bases de dados necessitam de softwares e tratamento estatístico adequado. Alguns pesquisadores, principalmente da área da saúde, possuem dificuldades no processo de extração e/ou análise desses dados, o que acaba inviabilizando ou desestimulando o uso dessas bases que representam fonte potencial e pouco explorada para o desenvolvimento de investigações científicas ${ }^{19,32}$.

\section{Limitações e obstáculos a serem superados}

A primeira etapa para utilização de dados secundários refere-se ao processo de extração dos microdados, que é realizado a partir dos sites já supracitados. A forma de extração e análise desses dados são temas pouco abordados nos currículos de pós-graduação dos cursos da área de saúde. Isso justifica o fato da maioria das pesquisas, que utilizam esses microdados, centrarem-se nas áreas de estatística e economia ${ }^{18,19}$.

Conforme sumarizado no Quadro 1, os inquéritos populacionais fornecem um acervo de informações sobre o estado nutricional e variáveis que podem ser determinantes do mesmo. Trata-se, portanto, de fonte potencial de informação para os profissionais de saúde e demais áreas desenvolverem pesquisas e discussões acerca das possibilidades de intervenções relacionadas à melhoria das condições de saúde da população.

Logo, destaca-se a importância dos cursos de pós-graduação em saúde pública, saúde coletiva e de nutrição desenvolverem disciplinas que abarquem a extração e utilização de bases de dados secundários, sendo que essas matérias poderiam contar com a participação de estatísticos e/ ou economistas a fim de aprimorar as discussões e ampliar o campo de oportunidades de desenvolvimento de estudos envolvendo diferentes profissionais e áreas de saberes ${ }^{18,19,32}$.

Além da extração, outra etapa importante refere-se à análise dos dados. Primeiramente, ressalta-se que os inquéritos populacionais, em função de restrições orçamentárias ou limitação de tempo para coleta dos dados, não utilizam processo de amostragem aleatória simples, mas sim o de amostragem estratificada ou por conglomerados, em múltiplos estágios, o que caracteriza um processo de amostragem complexo. Logo, as análises estatísticas de dados provenientes de amostras complexas são diferentes das tradicionais $^{32,33}$.

Ignorar o desenho amostral complexo durante as análises estatísticas pode gerar resultados distorcidos e comprometer as conclusões da pesquisa. Portanto, além de compreender o processo 
envolvido com a extração dos dados, se faz necessário o entendimento adequado para as análises estatísticas considerando o processo amostral complexo ${ }^{32,33}$.

Para extração e análise dos dados é importante a utilização de softwares que considerem o delineamento amostral complexo, sendo os mais utilizados: Stata, SAS e o R. Nesses softwares é possível incorporar, nas análises estatísticas, o efeito do desenho amostral complexo. Assim, torna-se importante a escolha adequada do software para realização da extração dos dados, bem como para realização das análises estatísticas ${ }^{32,33}$. Trabalhos que envolvem dados secundários, de inquéritos com desenho amostral complexo, devem deixar claro na metodologia que, para as análises estatísticas, considerou-se o delineamento complexo da amostra.

A escolha do software dependerá da facilidade de uso, do custo e dos recursos disponíveis de interesse para o pesquisador. Sendo importante a confirmação de que o software é indicado para análises de estudos com delineamentos complexos $^{33}$.

As dificuldades destacadas em relação à extração e à análise de dados secundários, dos inquéritos populacionais com plano de amostragem complexo, podem ser solucionadas com adequada formação e capacitação de pesquisadores e/ ou profissionais de diferentes formações acadêmicas. A qualificação e o aprimoramento profissional nessa área poderão formar pesquisadores com competência para desenvolver pesquisas de alta relevância para saúde individual e coletiva.

\section{Considerações finais}

O uso de dados secundários, disponibilizados pelos inquéritos populacionais brasileiros, constitui um frutífero campo de desenvolvimento de pesquisas. $\mathrm{O}$ acervo de variáveis disponíveis nas bases de dados dos inquéritos não é observado nos sistemas de informação em saúde, o que possibilita maior número de análises, determinação de fatores de exposição e controle para variáveis de confundimento. Isso reduz os vieses, aumenta a validade das pesquisas e a confiabilidade das conclusões.

No entanto, algumas limitações, como as relacionadas à extração e à análise dos dados, acabam por limitar sua utilização por pesquisadores e/ou profissionais da saúde, tornando-se importante a inclusão de disciplinas nos cursos de pósgraduação que impulsionem o uso dessas bases de dados. Seria interessante um trabalho conjunto de pesquisadores da saúde com economistas e estatísticos, que estão mais familiarizados em trabalhar com dados secundários de inquéritos populacionais.

Políticas públicas devem se embasar em evidências científicas produzidas por estudos com validade e precisão. A realização de inquéritos populacionais com produção de informações de relevância para saúde coletiva, assim como a qualificação profissional para utilização dessas informações, constituem meios de aprimoramento para o planejamento em saúde e elaboração de intervenções mais eficientes, eficazes e efetivas.

\section{Colaboradores}

N Sperandio participou da concepção, redação e revisão crítica do conteúdo intelectual e da aprovação da versão final do manuscrito. SE Priore contribuiu na revisão crítica do conteúdo e na aprovação da versão final do manuscrito. 


\section{Referências}

1. Viacava F. Informações em Saúde: a importância dos inquéritos populacionais. Cien Saude Colet 2002; 7(4):607-621.

2. Lopes ACS, Caiaffa WT, Mingoti SA, Lima-Costa MFF. Ingestão alimentar em estudos epidemiológicos. Rev Bras Epidemiol 2003; 6(3):209-219.

3. Batista Filho M, Rissin A. Nutritional transition in Brazil: geographic and temporal trends. Cad Saude Publica 2003; 19(1):181-91.

4. Instituto Brasileiro de Geografia e Estatística (IBGE). Estudo Nacional de Despesa Familiar - ENDEF. Dados preliminares. Consumo alimentar, antropometria. Rio de Janeiro: IBGE; 1977.

5. Instituto Brasileiro de Geografia e Estatística (IBGE). Pesquisa de Orçamentos Familiares 1987-1988: análise da disponibilidade domiciliar de alimentos. Rio de Janeiro: IBGE; 1999.

6. Instituto Brasileiro de Geografia e Estatística (IBGE). Pesquisa de Orçamentos Familiares 1995-1996: análise da disponibilidade domiciliar de alimentos. Rio de Janeiro: IBGE; 1997.

7. Instituto Brasileiro de Geografia e Estatística (IBGE). Pesquisa de Orçamentos Familiares 2002-2003: análise da disponibilidade domiciliar de alimentos e do estado nutricional no Brasil. Rio de Janeiro: IBGE; 204.

8. Instituto Brasileiro de Geografia e Estatística (IBGE). Pesquisa de Orçamento Familiares 208-2009: Análise do consumo alimentar pessoal no Brasil. Rio de Janeiro: IBGE; 2011.

9. Brasil. Ministério da Saúde (MS). Instituto Nacional de Alimentação e Nutrição (INAN). Pesquisa Nacional de sobre Saúde e Nutrição. Brasília: MS; 1990.

10. Instituto Brasileiro de Geografia e Estatística (IBGE). Pesquisa sobre Padrões de Vida-PPV, 1996-1997. Rio de Janeiro: IBGE; 1998.

11. Instituto Brasileiro de Geografia e Estatística (IBGE). Sociedade Civil Bem-Estar Familiar no Brasil. Pesquisa Nacional sobre Demografia e Saúde (PNDS), 1996. Rio de Janeiro: IBGE; 1996.

12. Brasil. Ministério da Saúde (MS). Departamento de Ciência e Tecnologia. Centro Brasileiro de Análise e Planejamento (CEBRAP). Pesquisa Nacional de Demografia e Saúde da Criança e da Mulher - PNDS 2006. Brasília: MS; 2008.

13. Instituto Brasileiro de Geografia e Estatística (IBGE). Pesquisa Nacional de Saúde, 2013. Rio de Janeiro: IBGE; 2014.

14. Instituto Brasileiro de Geografia e Estatística (IBGE). Pesquisa Nacional de Saúde do Escolar, 2009. Rio de Janeiro: IBGE; 2010.

15. Instituto Brasileiro de Geografia e Estatística (IBGE) Pesquisa Nacional de Saúde do Escolar, 2012. Rio de Janeiro: IBGE; 2013.

16. Vasconcellos MTL, Silva PLN, Szklo M, Kuschnir MCC, Klein CH, Abreu GA, Barufaldi LA, Bloch KV. Desenho da amostra do Estudo do Risco Cardiovascular em Adolescentes (ERICA). Cad Saude Publica 2015; 31(5):921-930.

17. Brasil. Ministério da Saúde (MS). Secretaria de Vigilância em Saúde. VIGITEL. Brasil 2013: Vigilância de fatores de risco e proteção para doenças crônicas por inquérito telefônico. Brasília: MS; 2014.

18. Coeli MC, Pinheiro RS, Carvalho MS. Nem melhor nem pior, apenas diferentes. Cad Saude Publica 2014: $30(7): 1-3$.
19. Szwarcwald CL, Damacena GN. Amostras complexas em inquéritos populacionais: planejamento e implicações na análise estatística dos dados. Rev Bras Epidemiol 2008; 11(Supl. 1):38-45.

20. Barra FBK, Lima RLEFP, Castro FSC, Priore SE. Instrumentos de inquérito dietético utilizados na avaliação do consumo alimentar em adolescentes: comparação entre métodos. ALAN 2007; 57(1):43-50.

21. Brasil. Ministério da Saúde (MS). Secretaria de Atenção à Saúde. Departamento de Atenção Básica. Coordenação Geral de Alimentação e Nutrição. Guia Alimentar para a população brasileira. Brasília: MS; 2014.

22. Levy-Costa RB, Claro RM, Mondini L, Sichieri R, Monteiro CA. Distribuição regional e socioeconômica da disponibilidade domiciliar de alimentos no Brasil em 2008-2009. Rev Saude Publica 2012; 46(1):6-15.

23. Martins APB, Levy RB, Claro RM, Moubarac JC, Monteiro CA. Participação crescente de produtos ultraprocessados na dieta brasileira (1987-2009). Rev Saude Publica 2013; 47(4):656-665.

24. Yokoo EM, Pereira RA, Veiga GV, Nascimento S, Costa RS, Marins VMR, Lobato JCP, Sichieri R. Proposta metodológica para o módulo de consumo alimentar pessoal na pesquisa brasileira de orçamentos familiares. Rev Nutr 2008; 21(6):767-776.

25. Conde WL, Oliveira DR, Borges CA, Baraldi LG. Consistência entre medidas antropométricas em inquéritos nacionais. Rev Saude Publica 2013; 47(1):69-76.

26. Schlickmann FDE, Adami F, GVFA, Altenburg AMA, Marino CMC, Kerpel R. Padronização e confiabilidade das medidas antropométricas para pesquisa populacional. ALAN 2007; 57(4):335-342.

27. Brasil. Ministério da Saúde (MS). Secretaria de Atenção à Saúde. Departamento de Atenção Básica. Protocolos do Sistema de Vigilância Alimentar e Nutricional - SISVAN na assistência à saúde. Brasília: MS; 2008.

28. Malta DC, Leal MC, Costa MFL, Morais NOL. Inquéritos Nacionais de Saúde: experiência acumulada e proposta para o inquérito de saúde brasileiro. Rev Bras Epidemiol 2008; 11(Supl. 1):159-167.

29. Coutinho JG, Cardoso AJC, Toral N, Silva ACF, Ubarana JA, Aquino KKNC, Nilson EAF, Fagundes A, Vasconcellos AB. A organização da Vigilância Alimentar e Nutricional no Sistema Único de Saúde: histórico e desafios atuais. Rev Bras Epidemiol 2009; 12(4):688-699.

30. Brasil. Ministério da Saúde (MS). Secretaria de Atenção à Saúde. Departamento de Atenção Básica. Política Nacional de Alimentação e Nutrição. Brasília: MS; 2012.

31. Coeli MC. Sistemas de Informação em Saúde e uso de dados secundários na pesquisa e avaliação em saúde. Cad Saude Colet 2010; 18(3):335-336.

32. Francisco PMSB, Donalisio MR, Barros MBA, Cesar CLG, Carandina L, Goldbaum M. Medidas de associação em estudo transversal com delineamento complexo: razão de chances e razão de prevalência. Rev Bras Epidemiol 2008; 11(3):347-355.

33. Kneipp SM, Yarandi HN. Complex sampling designs and statistical issues in secondary anlysis. West J Nurs Res 2002; 24(5):552-566.

Artigo apresentado em 04/11/2015

Aprovado em 10/05/2016

Versão final apresentada em 12/05/2016 